\title{
Electrostatic triple root structures: Connections to supersolitons, double layers, and acoustic speed solitons in nonthermal plasmas with negative and positive dust
}

\author{
Frank Verheest ${ }^{1,2}$ and Carel P. Olivier ${ }^{3}$ \\ ${ }^{1}$ Sterrenkundig Observatorium, Universiteit Ghent, Krijgslaan 281, B-9000 Ghent, Belgium \\ ${ }^{2}$ School of Physics, University of KwaZulu-Natal, Durban 4000, South Africa \\ ${ }^{3}$ Centre for Space Research, North-West University, Potchefstroom 2520, South Africa
}

(Received 21 September 2017; accepted 20 October 2017; published online 14 November 2017)

Large-amplitude dust-acoustic solitons and double layers in a dusty plasma model comprised of nonthermal Cairns electrons and ions, and cold, negative and positive dust grains had earlier been investigated, before newer concepts such as supersolitons, solitons at the acoustic speed, and even acoustic speed supersolitons arose. It turned out that under certain conditions, three distinct roots of the same polarity coalesce into a triple root, so that now a systematic investigation is carried out into the existence domains and properties of triple root solitary structures. From the analysis of the Sagdeev pseudopotentials, it transpires that there is a wide range of compositional parameters and soliton speeds where triple root structures can exist, and these are shown to be intimately connected with double layer and supersoliton ranges. Thus, triple roots are much more common than at first expected, even at the acoustic speed or in the presence of Boltzmann rather than Cairns nonthermal hot species. Once the existence domains are properly established, as many examples can be worked out as one chooses, generating typical soliton or double layer and electric field profiles. A great many combinations of compositional parameters and soliton speeds have been numerically tested, and they all reveal a similar pattern, with quantitative but no inherent qualitative changes. Published by AIP Publishing. https://doi.org/10.1063/1.5006026

\section{INTRODUCTION}

Dusty plasmas contain heavier grains, typically of micron size rather than on elementary particle scales, in the presence of traditional plasma electrons and ions. The plasma environment is responsible for the charging of these grains via different mechanisms, and it is no wonder that such mixtures occur in heliospheric and astrophysical plasmas, and also in laboratory and technological applications. ${ }^{1,2}$ In the commonly used charging model, the dust grains would be essentially charged by the capture of the more mobile electrons; hence, they become negatively charged. Lighter grains, however, might well be charged positively due to, e.g., photo-ionization or secondary electron emission, which is assumed to occur in the outer part of the rings of Saturn, in cometary tails, and in the Jovian magnetosphere. ${ }^{3-5}$

Because of the very small charge-to-mass ratio of the charged dust grains, the dust motion has to be taken into account at the lowest frequencies. Consequently, a new frequency range becomes important, below the ion-acoustic regime, of that of the dust-acoustic modes. ${ }^{6-9}$ As the coexistence of negative and positive dust has been observed in the Earth's magnetosphere and in cometary tails, ${ }^{4,5}$ there has been an interest in nonlinear waves in dusty plasmas containing both negatively and positively charged dust. ${ }^{10-15}$

We will revisit in this paper a specific dusty plasma model which includes nonthermal electrons and ions, and cold, negative and positive dust grains. This has been investigated already with an emphasis on the existence ranges and properties of dust-acoustic solitons and double layers,${ }^{16}$ before new concepts were introduced, such as supersolitons, ${ }^{17-25}$ solitons at the acoustic speed, ${ }^{26-31}$ and even acoustic speed supersolitons. ${ }^{32}$

In addition, for a very specific plasma composition and soliton speed, it was suggested that three roots of the same polarity might coalesce into a triple root. ${ }^{16}$ As we will show in what follows, the existence of triple root structures is intimately connected with double layer and supersoliton ranges. Such triple root structures hence call for a systematic investigation, rather than the incidental attention obtained so far, indicating that triple roots are more common than at first expected and can even occur at the acoustic speed or in the presence of Boltzmann rather than nonthermal hot species.

The paper is structured as follows: in Sec. II, we deal with the basic formalism, leading to the Sagdeev pseudopotential and its main properties; in Sec. III, the existence domains for negative triple root structures are determined, allowing then the generation of typical examples of Sagdeev pseudopotentials, and soliton and electric field profiles, including neighboring supersolitons and double layers; Sec. IV repeats this exercise for triple root structures at the acoustic speed, whereas in Sec. V, the Boltzmann limit for the hot species is discussed. Our conclusions are summarized in Sec. VI.

\section{BASIC FORMALISM}

As the specific dusty plasma model investigated in the present paper has been discussed already before, ${ }^{16}$ we very briefly repeat, in the interest of readability, some of the basic 
analysis needed to determine the Sagdeev pseudopotential ${ }^{33}$ for this composition. The model includes nonthermal electrons and ions, and cold, negative and positive dust grains. The fraction of charge residing on the negative dust is $f=Z_{d n} n_{d n 0} / n_{0}$ and on the electrons is $1-f=n_{e 0} / n_{0}$, whereas the fraction of charge residing on the positive dust is $g=Z_{d p}$ $n_{d p 0} / n_{0}$ and on the ions $1-g=n_{i 0} / n_{0}$. Here, $n_{0}=n_{e 0}+Z_{d n}$ $n_{d n 0}=n_{i 0}+Z_{d p} n_{d p 0}$, where $Z$ refers to the absolute value of the dust charges and the other symbols have their usual meaning.

In the Cairns nonthermal description, ${ }^{34}$ the normalized electron and ion densities are given by

$$
\begin{aligned}
& n_{e}=(1-f)\left(1-\beta \varphi+\beta \varphi^{2}\right) \exp [\varphi], \\
& n_{i}=(1-g)\left(1+\beta \varphi+\beta \varphi^{2}\right) \exp [-\varphi],
\end{aligned}
$$

respectively, in terms of an electrostatic potential $\varphi$ normalized to $\kappa T / e$ and of the macroscopic nonthermality parameter $\beta$. For reasons explained elsewhere, ${ }^{16}$ the same value of $\beta$ and $T$ is used for the electrons and the ions.

The cold dust is described by the continuity and momentum equations in normalized variables, ${ }^{26,27}$ referred, among others, to a measure for the dust-acoustic speed $c_{d a}=\left(Z_{d n} \kappa T / m_{d n}\right)^{1 / 2}$. The normalization implies that the space coordinate is in units of $\left(\varepsilon_{0} \kappa T / n_{0} e^{2}\right)^{1 / 2}$, and the negative dust species is the reference, leading to a normalized mass-per-charge $\mu=m_{d p}$ $Z_{d n} / Z_{d p} m_{d n}$.

If the common charging models are adopted, the heavier dust is negatively charged due to primary electron capture and the lighter dust might then be positive, due to, e.g., photo-ionization, in which case $\mu<1$. However, evaluation of models where $\mu=1$ or $\mu>1$ is perfectly covered by our formalism, as will be seen below.

In a frame where the nonlinear structure is stationary $(\partial / \partial t=0)$ and all variables tend to their undisturbed values at $x \rightarrow-\infty$, one derives that the normalized dust densities are given by

$$
n_{d n}=\frac{f}{\sqrt{1+\frac{2 \varphi}{M^{2}}}}, \quad n_{d p}=\frac{g}{\sqrt{1-\frac{2 \varphi}{\mu M^{2}}}},
$$

in terms of the normalized soliton velocity $M=V / c_{d a}$, with $V$ being the physical velocity of the solitary structure in an inertial frame.

There are limitations on $\varphi$, on the negative side at $\varphi_{n}=-M^{2} / 2$ and on the positive side at $\varphi_{p}=\mu M^{2} / 2$. If one of the dust species is totally absent, ${ }^{26,27}$ there are limits only on one side, but on the other side, double layers are possible under favourable circumstances, at near depletion of the plasma species of the same sign as the dust.

The basic set of equations is closed by the Poisson's equation, which gives after integration an energy-like integral

$$
\frac{1}{2}\left(\frac{d \varphi}{d x}\right)^{2}+S(\varphi, M)=0,
$$

in terms of a Sagdeev pseudopotential

$$
\begin{aligned}
S(\varphi, M)= & f M^{2}\left(1-\sqrt{1+\frac{2 \varphi}{M^{2}}}\right)+(1-f) \\
& \times\left\{1+3 \beta-\left(1+3 \beta-3 \beta \varphi+\beta \varphi^{2}\right) \exp [\varphi]\right\} \\
& +g \mu M^{2}\left(1-\sqrt{1-\frac{2 \varphi}{\mu M^{2}}}\right)+(1-g) \\
& \times\left\{1+3 \beta-\left(1+3 \beta+3 \beta \varphi+\beta \varphi^{2}\right) \exp [-\varphi]\right\}
\end{aligned}
$$

For brevity, compositional parameters like $f, g, \beta$, and $\mu$ are not explicitly written as arguments of $S(\varphi, M)$, whereas $M$ contains the as yet unknown soliton velocity $V$ and the main variable is the electrostatic potential $\varphi$. By construction and assumption $S(0, M)=S^{\prime}(0, M)=0$, but to obtain solitary waves $S^{\prime \prime}(0, M) \leq 0$ is needed, so that the origin is a (local) unstable maximum, at least on one side if not on both $\varphi$ sides. Derivatives of $S(\varphi, M)$ with respect to $\varphi$ are denoted by primes.

The convexity condition, $S^{\prime \prime}(0, M) \leq 0$, ensures that the nonlinear structures are superacoustic or, exceptionally, occur at the global acoustic speed, and $S^{\prime \prime}(0, M)=0$ yields the minimal $M$ for their existence

$$
M^{2} \geq M_{s}^{2}=\frac{f \mu+g}{(1-\beta) \mu(2-f-g)} .
$$

Here, $M_{s}$ is a measure for the global acoustic speed, in terms of the chosen normalization, and $M / M_{S}$ is then the true Mach number of the solitary structures, because the normalization of the velocities cancels out.

Moreover, $S^{\prime}(\varphi, M)$ represents in normalized parameters the total charge density, involving the expressions (1) and (2) with the appropriate signs, and it is seen that $\lim _{\varphi \rightarrow \varphi_{n, p}} S^{\prime}(\varphi, M) \rightarrow \mp \infty$. Owing to the required convexity to obtain solitary waves, $S^{\prime}(\varphi, M)$ goes through $\varphi=0$ with a negative or zero slope, and hence $S^{\prime}(\varphi, M)$ has an odd number of positive and negative zeroes, outside $\varphi=0$, before the asymptotes at $\varphi_{n, p}$ are reached, whereas $S(\varphi, M)$ is well behaved for all accessible $\varphi$.

The zeroes of $S^{\prime}(\varphi, M)$ are charge-neutral points, by definition, and they are a necessary condition to obtain roots for $S(\varphi, M) \cdot{ }^{35,36}$ Unfortunately, they are not sufficient to indeed obtain soliton or double layer roots. Single roots give the usual hump- or dip-like soliton, if there is only one charge-neutral point between it and the undisturbed condition at $\varphi=0$, or supersolitons if there are three distinct intermediate charge neutral points. The latter then correspond to three local extrema of the same polarity, where $S(\varphi, M)<0$, so that the supersoliton root is the first accessible one.

On the contrary, a double root/layer also counts as a charge-neutral point, with an intervening local maximum where $S(\varphi, M)<0$. A triple root structure corresponds to two coinciding charge-neutral points, and, as we will see below, behaves as a double layer of a rather peculiar kind. To avoid the longish expression "triple root double layer," we have used a "triple root structure." 
There is a link between the sign of

$$
S^{\prime \prime \prime}\left(0, M_{s}\right)=f-g+\frac{3(1-\beta)^{2}(2-f-g)^{2}\left(g-f \mu^{2}\right)}{(f \mu+g)^{2}},
$$

and the polarity of Korteweg-de Vries (KdV)-like solitons. ${ }^{29,30,37,38}$ By KdV-like, we mean that solitons share the property of the weakly nonlinear solitons which are solutions of a KdV equation, in that their amplitude shrinks and can be arbitrarily small when $M \rightarrow M_{s}$ from above. Such KdV-like solitons are thus superacoustic, but in the Sagdeev formalism there is no need to restrict the soliton amplitudes a priori, in the sense that a reductive perturbation limitation to quadratic or cubic nonlinearities requires them to be. On the contrary, the amplitudes in Sagdeev pseudopotential treatments are limited by physical constraints, and not by mathematical considerations.

In the case of coexistence of solitons of negative and of positive polarity, one polarity generates $\mathrm{KdV}$-like solitons, and the other what has been called non-KdV-like solitons. ${ }^{29,30,37,38}$ The latter are characterized by a finite amplitude at $M=M_{s}$, and hence are linked to the existence of solitons at the acoustic speed. Since the sign of $S^{\prime \prime \prime}\left(0, M_{s}\right)$ determines the polarity of the $\mathrm{KdV}$-like solitons, the nonKdV-like solitons have the opposite polarity. By "coexistence" we mean that solutions of both polarities can exist for a given set of compositional and speed parameters, but it is understood that only one solitary wave or double layer can be realized at a time, much like for an unstable equilibrium in one-dimensional motion, the particle can only slide down the hill in one direction at a time.

Finally, we mention a symmetry in the Sagdeev pseudopotential (4). It is easily verified that the Sagdeev pseudopotential is invariant under the simultaneous substitutions

$$
\varphi \rightarrow-\varphi, \quad f \leftrightarrow g, \quad \mu \rightarrow \frac{1}{\mu}, \quad M \rightarrow \sqrt{\mu} M .
$$

\section{TRIPLE ROOTS, DOUBLE LAYERS, AND SUPERSOLITONS}

Given that the earlier paper ${ }^{16}$ already gave a discussion of different dust-acoustic solitons and double layers in the chosen plasma model, we will focus here on the aspects which were not covered: triple root structures, and, in particular, how the triple root range is connected with double layers and supersolitons. In the Secs. IV and V, we will address triple root structures at the acoustic speed and also in the Boltzmann limit for the hot species.

It should be noted that, because of the symmetry (7), a negative triple root for $\mu>1$ corresponds to a positive triple root for $\mu<1$. With this in mind, we do not place any limits on the choice of $\mu$, and we can restrict the complete analysis to (nonlinear) modes of one polarity. We have chosen to investigate the properties of negative triple root structures and related solitons and double layers. However, some of the triple root structures might happen to coexist in certain parameter regimes with modes of the other, positive polarity. We will encounter some of those and include them on the graphs where useful, although they are not the main focus of the paper. All the conclusions automatically also hold for the positive $\varphi$ range, by simple mirroring of the relevant graphs in $\varphi$, with judicious adjustments of the normalization.

\section{A. Existence domains for negative triple roots}

We start with the existence domains of negative triple roots, the conditions for which being that

$$
S(\varphi, M)=0, \quad S^{\prime}(\varphi, M)=0, \quad S^{\prime \prime}(\varphi, M)=0
$$

should have a common root $\varphi \neq 0$ outside equilibrium. There are four compositional parameters, $f, g, \beta$, and $\mu$, plus the solitary structure speed $M$, which needs to be determined.

To investigate the dependence of the triple root structures on these parameters, we take a typical $f$ and pick some representative $g$, so that (8) determines $M, \beta$, and $\varphi$, as functions of $\mu$. These existence domains cover quite a range of parameter values, but it is seen in Figs. 1-3, which are for $f=0.3,0.5$, and 0.7 , respectively, that these result only in quantitative changes, with the overall qualitative trends being very similar. Thus, $M / M_{s}, \beta$, and the triple root amplitudes $|\varphi|$ increase with $\mu$, although at high $g$ the amplitude is nearly constant. Initially, as $\mu$ increases, Sagdeev pseudopotentials also have positive roots for the same compositional parameters, but these are not otherwise considered here, except that their amplitudes have been shown in Figs. $1(\mathrm{c})-3(\mathrm{c})$.

In those figures, the lower limits on $\mu$ are given by $M / M_{s}$ starting from $M / M_{s}=1$. The upper limits on $\beta$ have been set at $\beta_{\ell}=4 / 7$, because for larger $\beta$ the underlying phase space Cairns distribution has no longer only one local maximum but develops wings, and thus two-beam instabilities might arise. ${ }^{26}$ This cut-off at $\beta_{\ell}=4 / 7$ indicates how far one can go in the range $\mu>1$, except in Fig. 3, where one can seemingly go to very high values for $\mu$, but for graphical clarity, the curves have been limited to $\mu=10$.

On the contrary, the limit for $f=0.3$ is near $\mu=1.68$ for $g=0.9$, but at smaller $\mu$ for $g<0.9$, whereas for $f=0.5$ it is at $\mu=5$ for $g=0.9$. The graphs also indicate that $g>f$ is needed to have triple root structures.

At $f=0.3$, the negative solitons and triple root structures are $\mathrm{KdV}$-like below and non-KdV-like above $g=0.8$, with a corresponding crossover at $\beta=0.386$. Conversely, the positive solitons are $\mathrm{KdV}$-like above and non-KdV-like below $g=0.8$, which can be seen in the positive range for $\varphi$ in Fig. 1. We remark here that for $g=0.4$ and $g=0.5$, values of $\beta>\beta_{\ell}=4 / 7$ are needed to generate triple root structures, and hence, the corresponding curves have been omitted.

At $f=0.5$, the negative solitons and triple root structures are KdV-like below and non-KdV-like above $g=0.723$, with a corresponding crossover at $\beta=0.312$. Conversely, the positive solitons are KdV-like above and non-KdV-like below $g=0.723$, as seen in Fig. 2(c) on the positive range for $\varphi$.

For $f=0.7$, the negative solitons and triple root structures are KdV-like and the positive solitons are non-KdVlike for the whole range, without crossover, as seen in Fig. 3. As mentioned already, there is no obvious limit to $\mu$. 

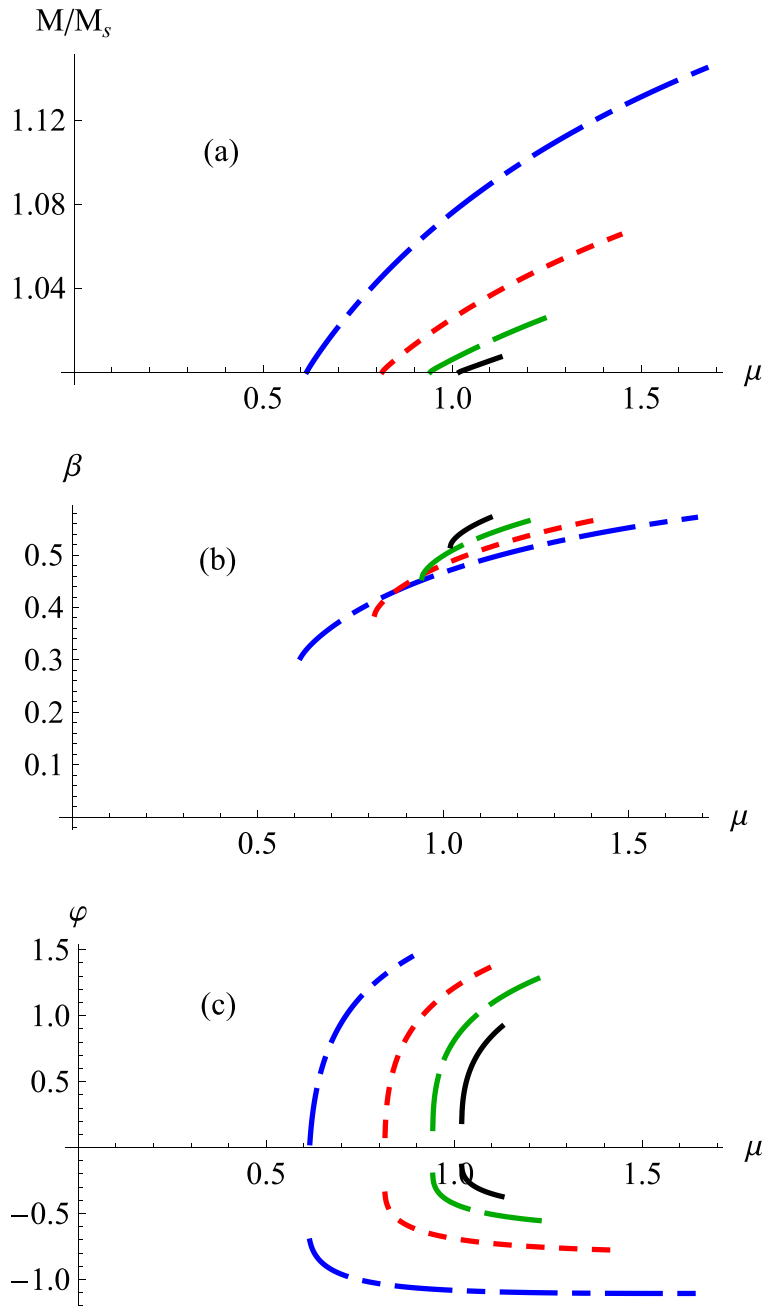

FIG. 1. Existence domains for negative triple root waves, drawn as functions of $\mu$, for $f=0.3$ but different $g$, for (a) Mach numbers $M / M_{s}$, (b) $\beta$, and (c) corresponding triple root amplitudes $\varphi$. The curve coding is for $g=0.6$ (black solid), $g=0.7$ (green dashed), $g=0.8$ (red dotted), and $g=0.9$ (blue dotted-dashed). In (c), the positive roots of some of the Sagdeev pseudopotentials with negative triple structures have also been shown, but their existence is limited at an increasing amplitude by $\varphi_{p}$, encountered at $\mu=1.232$ for $g=0.7$, at $\mu=1.104$ for $g=0.8$, and at $\mu=0.886$ for $g=0.9$. For $g=0.6$, the imposed limit $\beta_{\ell}=4 / 7$ intervenes before $\varphi_{p}$ is reached.

Because the Sagdeev pseudopotential is, from a mathematical point of view, continuous in the different compositional parameters, Mach numbers, and the electrostatic potential, there is a wide domain where triple root structures can exist. Once the existence domains are established, one can work out as many examples in detail as one chooses. We have tested a great many combinations of compositional parameters, and they all reveal a similar pattern, with quantitative but no qualitative changes. None of these are included here, to avoid overloading the paper with repetitive graphs and figures, except for one typical example, selected among an infinity of other equally valid choices with analogous results. This is worked out in Sec. III B

\section{B. Sagdeev pseudopotentials, soliton, and electric field profiles}

As a generic illustration, we pick $f=0.5, g=0.8$, $\mu=1$, and $\beta=0.358$, where the $\beta$ value ensures that a triple
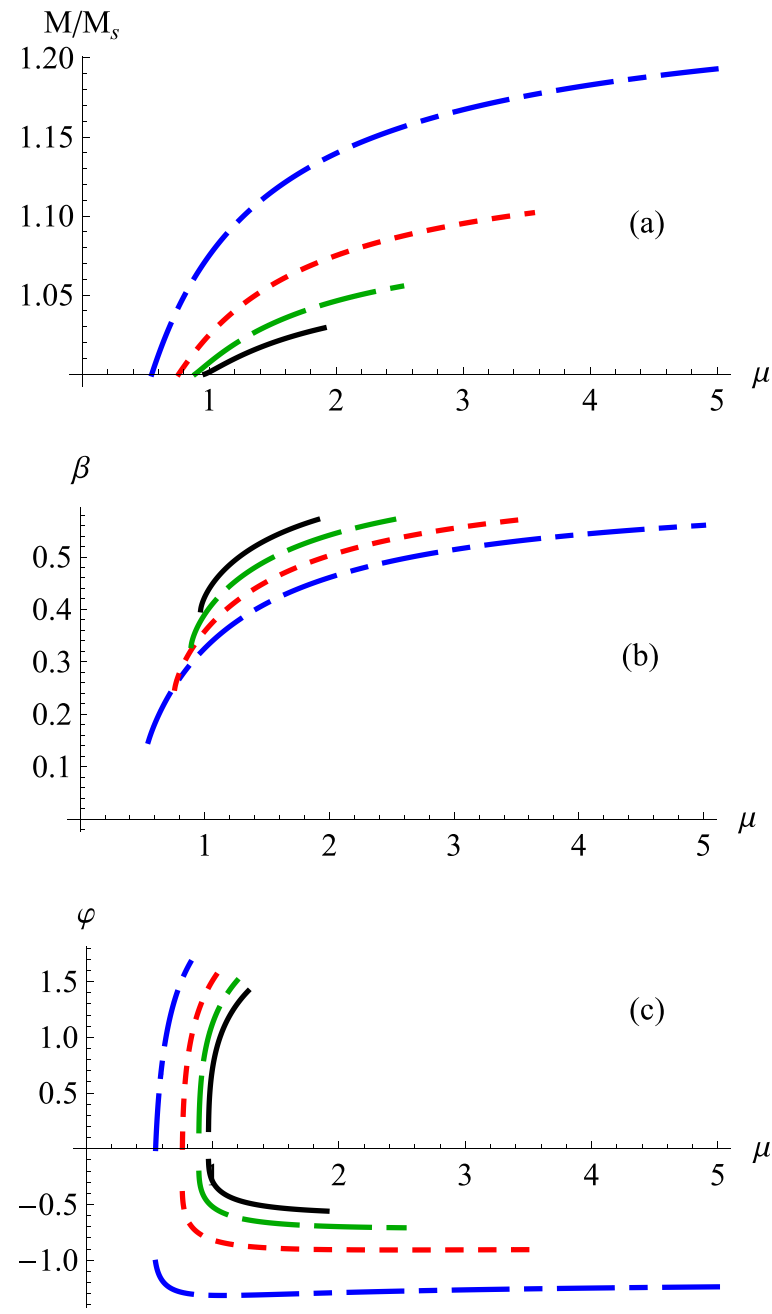

FIG. 2. Existence domains for $f=0.5$ with the same coding and interpretation as in Fig. 1. The positive root amplitude is limited by $\varphi_{p}$, encountered at $\mu=1.279$ for $g=0.6$, at $\mu=1.192$ for $g=0.7$, at $\mu=1.056$ for $g=0.8$, and at $\mu=0.839$ for $g=0.9$.

root exists, at $\varphi=-0.818$ for $M / M_{s}=1.0248$, and the corresponding Sagdeev pseudopotential is indicated in Fig. 4 by the solid blue line. Taking a lower value $M / M_{s}=1.0233$ gives a Sagdeev pseudopotential with a normal soliton root at $\varphi=-0.535$ (long green dashes), whereas a higher value $M / M_{s}=1.0252$ gives a supersoliton root at $\varphi=-0.988($ red dashes), all for exactly the same plasma composition.

The corresponding solitary structure profiles and electric fields are shown in Fig. 5, for the same parameter values and curve coding. The solid blue line shows the triple root structure. It follows that the triple root serves as the end of a range of solitons, at increasing $M / M_{s}$, and for larger $M / M_{s}$ a range of supersolitons occurs, until the telltale electric field wiggles disappear.

Next, we focus on changes in $\beta$, leaving the other compositional parameters unchanged. First, when $\beta$ decreases below the value needed to obtain a triple root structure, we cannot find double layers, but there is a small range where supersolitons can exist. This is illustrated in Fig. 6, for the same parameters $f=0.5, g=0.8$, and $\mu=1$, but $\beta=0.356$. The green dashed curve signals a Sagdeev pseudopotential with an inflection point, where two of the local extrema have 

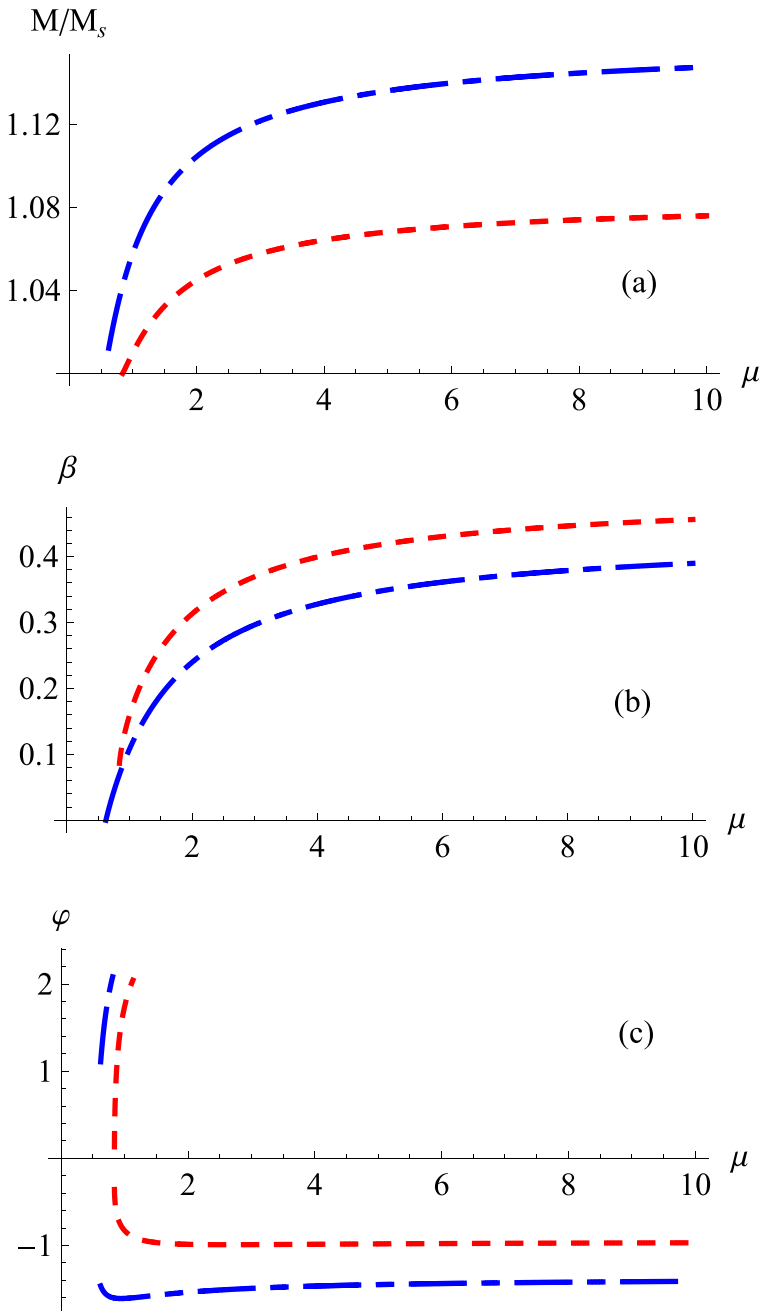

FIG. 3. Existence domains for $f=0.7$ with the same coding and interpretation as in Fig. 1. The positive root amplitudes are limited by $\varphi_{p}$, encountered at $\mu=1.13$ for $g=0.8$, and at $\mu=0.886$ for $g=0.9$.

coalesced, and for larger $M / M_{s}$ we find supersoliton curves. When $\beta$ increases towards the triple root value, the inflection point gets closer to the horizontal axis, until a triple root is generated.

On the contrary, we note that at increasing $\beta>0.358$, other plasma parameters being equal, the triple root signals the beginning of a range of double layers as limiting factors of a range of ordinary solitons. To illustrate this, we pick

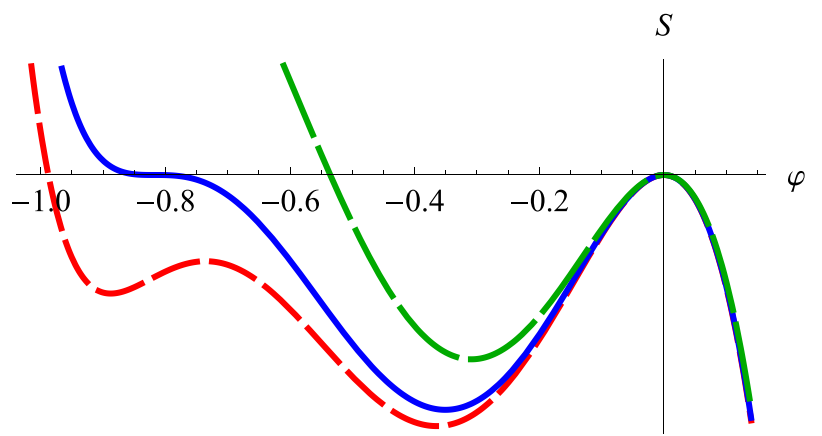

FIG. 4. Typical Sagdeev pseudopotentials having a negative triple root structure (blue curve, $M / M_{s}=1.0248$ ), a supersoliton (red dashes, $M /$ $M_{s}=1.0252$ ), and a standard soliton (long green dashes, $M / M_{s}=1.0233$ ), all three for $f=0.5, g=0.8, \mu=1$, and $\beta=0.358$.
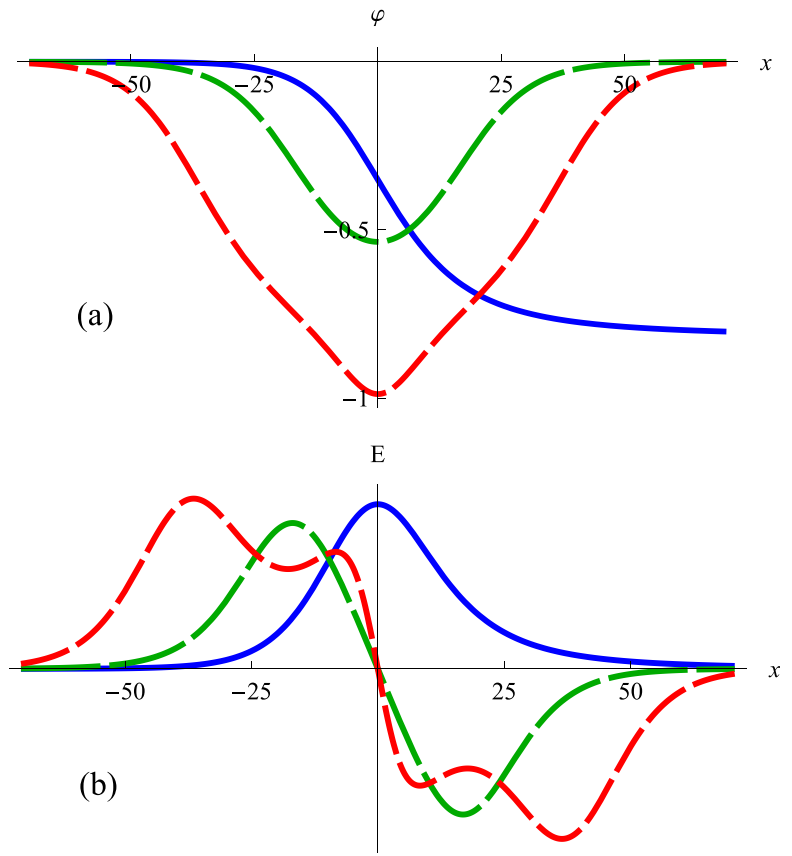

FIG. 5. (a) Solitary structure profiles and (b) electric fields are plotted for the Sagdeev pseudopotentials shown in Fig. 4, for the same parameter values and curve coding.

$\beta=0.36$ and plot the results in Fig. 7, with the same curve and parameter conventions as in Fig. 4, except that the blue curve now refers to the double layer rather than the triple root structure. Here, the double layer ends a range of standard solitons, beyond which a range of supersolitons exist, all for increasing $M / M_{s}$. The inflection point is now shifted above the horizontal axis, as shown by the green dashed curve. It turns out that for the plasma parameters chosen to illustrate this subsection, once $\beta>0.358$, one can increase $\beta$ up to $\beta_{\ell}$ and still find double layers. The supersoliton range ends, however, when the third negative root of the Sagdeev pseudopotential no longer exists, i.e., once $\varphi_{n}$ is reached.

The corresponding solitary structure profiles and electric fields are shown in Figs. 8(a) and 8(b), respectively, for the same parameter values and curve coding.

Although it is difficult to see on the solid blue graphs in Figs. 5 and 8 , there is a distinct difference between the triple and the double root profiles, in the way they approach zero or the triple/double root for large $x$. At $\varphi=0$, as also for a

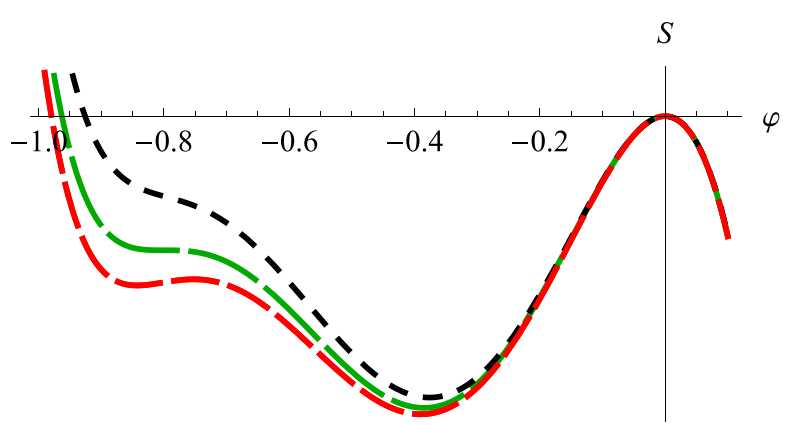

FIG. 6. Typical Sagdeev pseudopotentials having a negative supersoliton (red dashes, $M / M_{s}=1.0259$ ) and two ordinary solitons (long green dashes, $M / M_{s}=1.0258$ and black dots, $\left.M / M_{s}=1.0256\right)$, all three for $f=0.5, g=0.8$, $\mu=1$, and $\beta=0.356$. 


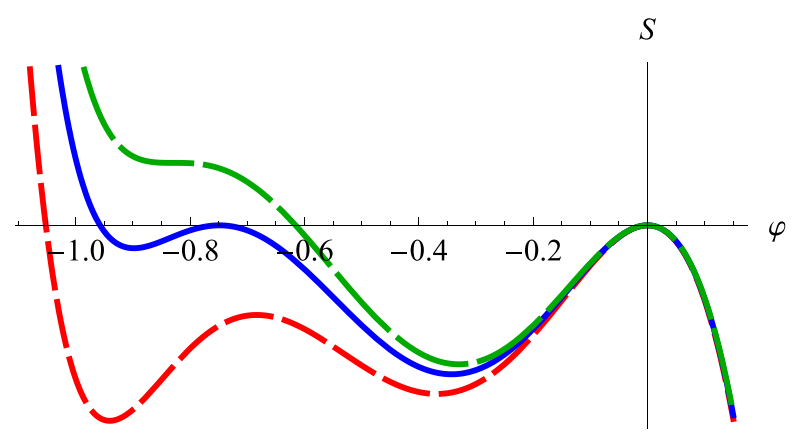

FIG. 7. Typical Sagdeev pseudopotentials having a negative double layer (blue curve, $M / M_{s}=1.0245$ ), a supersoliton (red dashes, $M / M_{s}=1.0252$ ), and a standard soliton (long green dashes, $M / M_{s}=1.0241$ ), all three for $f=0.5, g=0.8, \mu=1$, and $\beta=0.36$.

double layer, $S=0$ and $S^{\prime}=0$, so that the decay of the profile is exponential for large $|x| .{ }^{39}$ On the contrary, the (negative) triple root conditions are $S=0, S^{\prime}=0$, and $S^{\prime \prime}=0$, for a value $\varphi<0$, which implies that the decay is algebraic for $x \rightarrow+\infty$, whereas it remains exponential for $x \rightarrow-\infty$. $^{39}$

This is illustrated in more detail in Fig. 9, where we focus on the typical triple root structure (blue solid curve, copied from 5) and on the double layer (red dashed curve) profiles having the same amplitude $\varphi=-0.818$ and for the same $f=0.5, \mu=1$, and $\beta=0.358$. However, one cannot have all compositional parameters equal, so that $g=0.8$ and $M / M_{s}=1.0248$ yield a triple root structure and $g=0.856$ and $M / M_{s}=1.0430$ a double layer. On the approach $x \rightarrow-\infty$ both curves are very similar, but there is quite a distinction between exponential and algebraic approaches for $x \rightarrow+\infty$.

Similar effects can be noted upon the perusal of the solitary structure profiles in Figs. 5 and 8, but are there harder to see clearly.
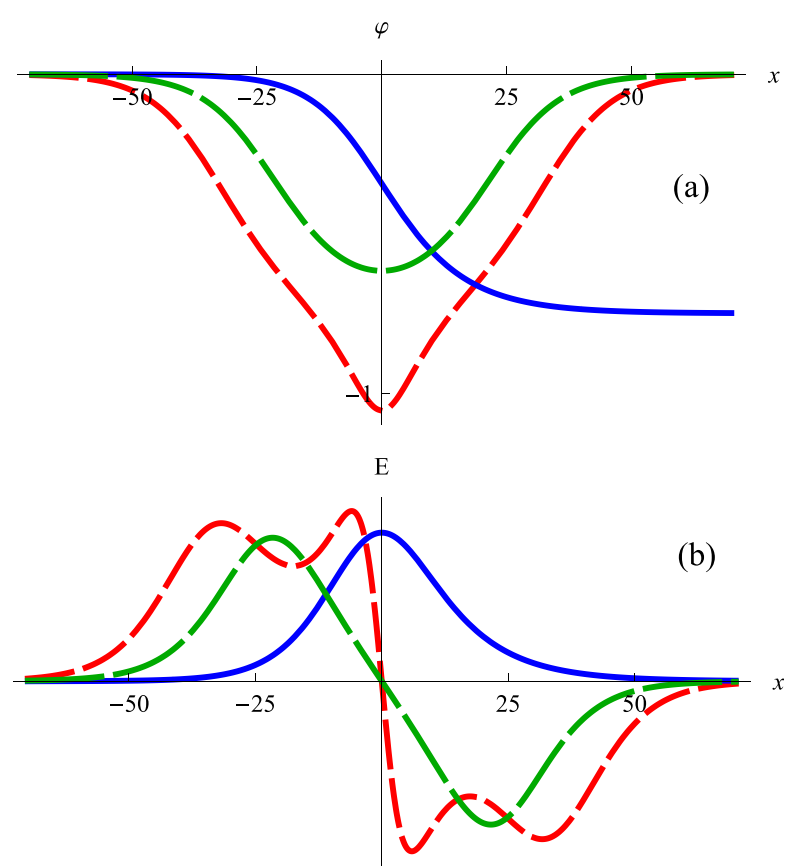

FIG. 8. (a) Solitary structure profiles and (b) electric fields corresponding to the Sagdeev pseudopotentials shown in Fig. 7, for the same parameter values and curve coding.
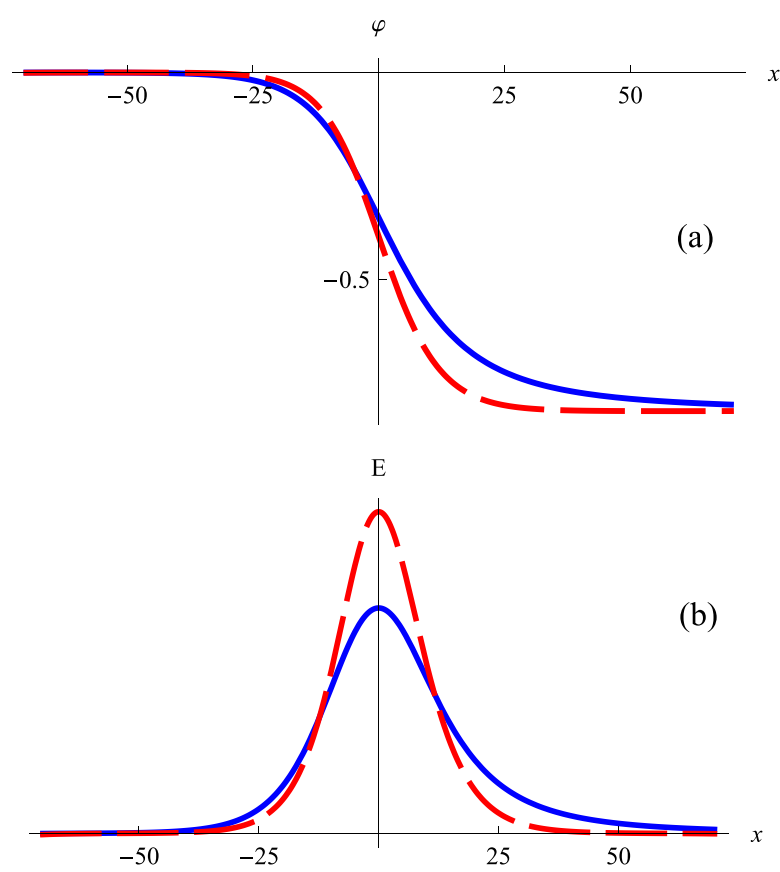

FIG. 9. Focus on the approach $x \rightarrow+\infty$ of typical triple root (blue solid curve) and double layer (red dashed curve) profiles (a) and electric fields (b), having the same amplitude $\varphi=-0.818$, for $f=0.5, \mu=1$, and $\beta=0.358$, but slightly different $g$ and $M / M_{s}$. These are $g=0.8$ and $M / M_{s}=1.0248$ for the triple root and $g=0.856$ and $M / M_{s}=1.043$ for the double layer.

\section{ACOUSTIC SPEED SOLITONS}

Remarkably (at least it came as a surprise to us), there are even triple root structures at the acoustic speed. Because at the acoustic speed $S\left(\varphi, M_{S}\right)$ has a triple root at the origin for the undisturbed conditions $(\varphi=0)$, Sagdeev pseudopotentials with a triple root $\varphi \neq 0$ have to be negative between those two triple roots.

Before giving an example, we have investigated the possibilities of finding solutions to

$$
S\left(\varphi, M_{s}\right)=0, \quad S^{\prime}\left(\varphi, M_{s}\right)=0, \quad S^{\prime \prime}\left(\varphi, M_{s}\right)=0,
$$

where effectively $M$ has disappeared from the set. There remain in (9) four compositional parameters, $f, g, \beta$, and $\mu$. Following a variant of the method used in Sec. III, we pick some values for $f$, and let (9) determine $g, \beta$, and $\varphi$ as functions of variable $\mu$. This gives the typical existence curves shown in Fig. 10, but other, equivalent procedures are possible. It shows that as $\mu$ increases, at a given $f, \beta$ increases, but $g$ and the amplitudes $|\varphi|$ decrease.

The curves for $g$ and $\varphi$ show little variation with $f$ or $\beta$, and because $|\varphi|$ becomes very small near $\mu=1$, that signals the end of the ranges. We have been unable to find such triple roots at the acoustic speed for $f \geq 0.8$, which can be guessed from Fig. 10(b). As in Sec. III, the solitons are all negative, but now $S^{\prime \prime \prime}\left(0, M_{S}\right)>0$, so that the triple root and other solitons are non-KdV-like.

To illustrate our findings (again for one generic example to avoid tedious repetitions with analogous characteristics), given that we have quite a choice we pick $f=0.3$ and $g=0.9$ and then $\mu=0.615$ and $\beta=0.3032$ ensure that a triple root exists, at $\varphi=-0.697$. The corresponding Sagdeev 

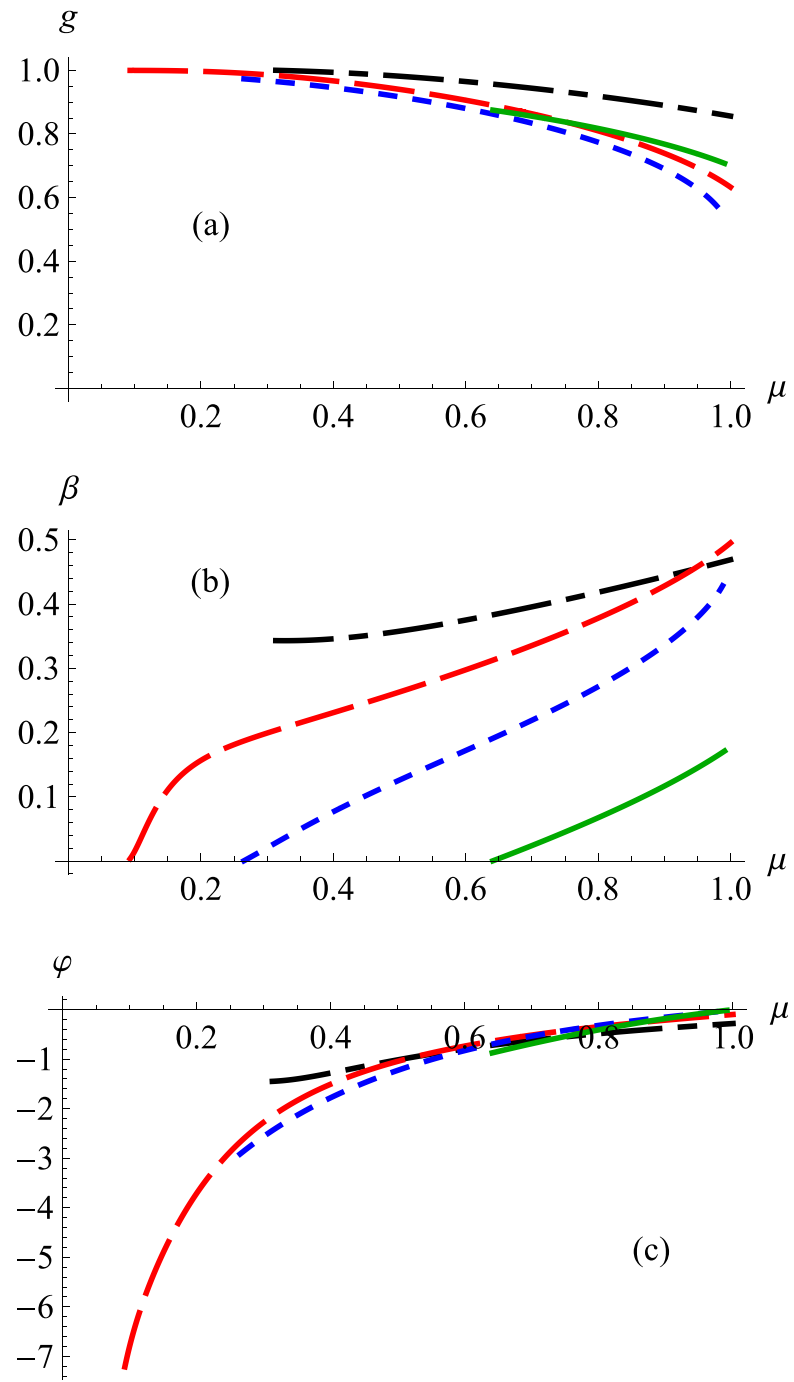

FIG. 10. Existence domains for negative triple root structures at the acoustic speed, drawn as functions of $\mu$, for different values of $f$, and (a) $g$, (b) $\beta$, and (c) corresponding triple root structure amplitudes $\varphi$. The curve coding is for $f=0.1$ (black dotted-dashed), $f=0.3$ (red dashed), $f=0.5$ (blue dotted), and $f=0.7$ (green). The curves are limited by the approach $\mu \rightarrow 1$.

pseudopotential is indicated in Fig. 11 by the solid blue line. Since the negative triple root is at the acoustic speed, only negative superacoustic non-KdV-like modes are possible. By varying $\beta$, one obtains neighboring supersolitons, all at the acoustic speed $\left(M / M_{s}=1\right)$.

We remark that the Sagdeev pseudopotential with a triple root structure has two triple roots: because of it being at the acoustic speed, one is at the origin, and the other is at the root itself. As there are two consecutive triple roots, the soliton and electric field profiles will decay algebraically on both sides. ${ }^{39}$ Since the graphs of these otherwise look very similar to those of Figs. 5 and 8, they have not been included here.

We have not specifically looked for acoustic speed supersolitons, since these have already been found and discussed at length in an earlier paper. ${ }^{32}$ This was based on a simplification of the model investigated here, where one of the nonthermal hot species had been omitted. In the more complicated plasma composition we are investigating in the present paper, having more leeway in the hot species density

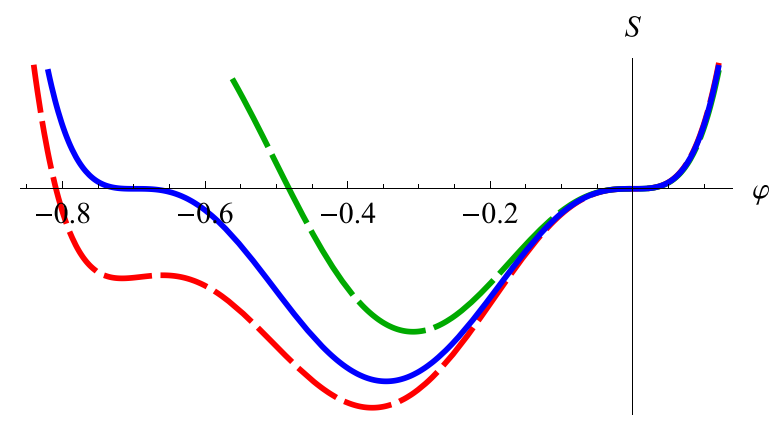

FIG. 11. Typical Sagdeev pseudopotentials having a negative triple root structure at the acoustic speed (blue curve, for $\beta=0.3032$ ), a supersoliton (red dashes, for $\beta=0.3029$ ), and an ordinary soliton (long green dashes, for $\beta=0.304)$, for $f=0.3, g=0.9$, and $\mu=0.615$.

parameters, acoustic speed supersolitons certainly exist here, but we have shied away from a tedious exploration of this, the emphasis being on triple root structures. And as is obvious from the Sagdeev pseudopotential triple root curves, triple roots can initiate a range of supersolitons, as in other circumstances double roots can do, but the triple roots cannot themselves be supersolitons.

Trying to see if there are neighboring double layers at the acoustic speed (an aspect not studied in the earlier paper ${ }^{16}$ ) by modifying $\beta$ and $\mu$, we could find much weaker double layers and associated supersolitons. A typical example is shown in Fig. 12, for a Sagdeev pseudopotential having a negative double root structure at the acoustic speed (blue curve, for $\beta=0.3145$ ) and a supersoliton (red dashes, for $\beta=0.3142$ ), both for $f=0.3, g=0.9$ and $\mu=0.6$. In (a) the full curves are shown, up to the supersoliton root at
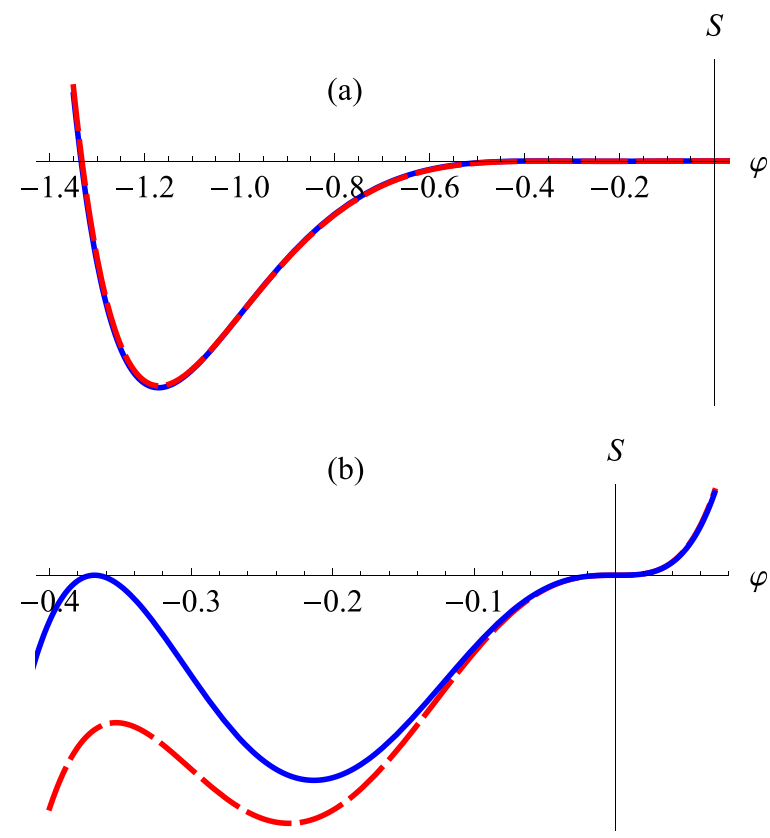

FIG. 12. Typical Sagdeev pseudopotentials having a negative double root structure at the acoustic speed (blue curve, for $\beta=0.3145$ ) and a supersoliton (red dashes, for $\beta=0.3142$ ), both for $f=0.3, g=0.9$, and $\mu=0.6$. In (a), the full curves are shown up to the supersoliton root at $\varphi=-1.332$, near a nonaccessible root $\varphi=-1.334$ of the double layer Sagdeev pseudopotential. As the double layer at $\varphi=-0.368$ cannot be seen on the scale of (a), a blowup is shown in (b). 
$\varphi=-1.332$, near a nonaccessible root $\varphi=-1.334$ of the double layer Sagdeev pseudopotential. As the double layer at $\varphi=-0.368$ cannot be seen on the scale of (a), a blowup concentrating on it is shown in (b).

Because of the character of the acoustic speed double layer, the origin corresponds to a triple root, to which the double layer amplitude decays algebraically, whereas at the double layer itself the decay is exponential. ${ }^{39}$

\section{BOLTZMANN LIMIT}

One can note on the curves of Fig. 10(b) that for $f=0.3$, $f=0.5$, and $f=0.7$, acoustic speed triple root structures can be found even when $\beta=0$, i.e., the Boltzmann limit. To study the situation in the Boltzmann limit for general triple root structures and their associated supersolitons, we put in (4) and (8) $\beta=0$ and are left with the parameters and variables $f, g, \mu, M$, and $\varphi$. As $g$ shows little variation, we typically take $g=0.9$ and determine from (8) $M, f$ and $\varphi$, as functions of $\mu$. This is illustrated in Fig. 13.

To illustrate our findings (again for one generic example to avoid tedious repetitions with analogous characteristics),
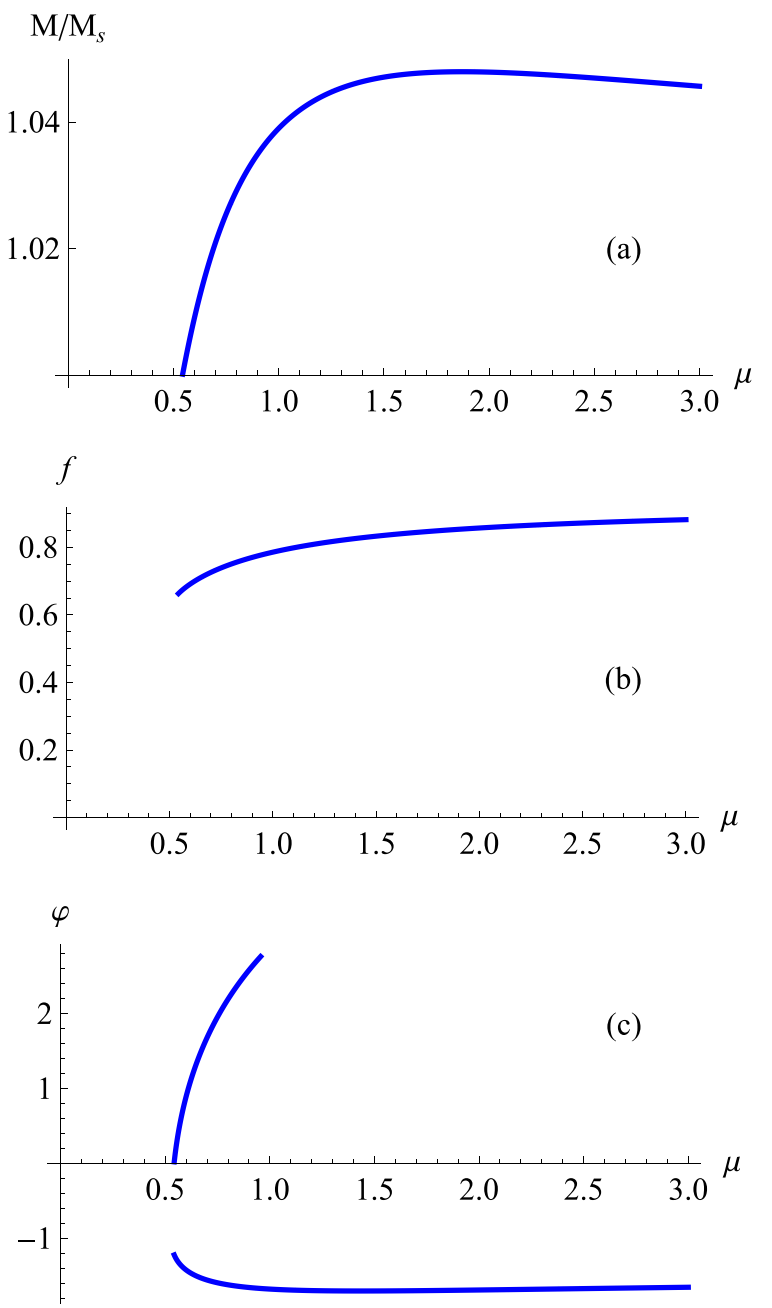

FIG. 13. Existence domains for negative triple root waves, drawn as functions of $\mu$, for a typical $g=0.9$ and in the Boltzmann limit $\beta=0$, for (a) Mach numbers $M / M_{s}$, (b) $f$, and (c) triple root amplitudes $\varphi$. In (c), the positive roots have also been shown, but the existence of these is limited at increasing amplitude by $\varphi_{p}$, encountered at $\mu=0.955$.

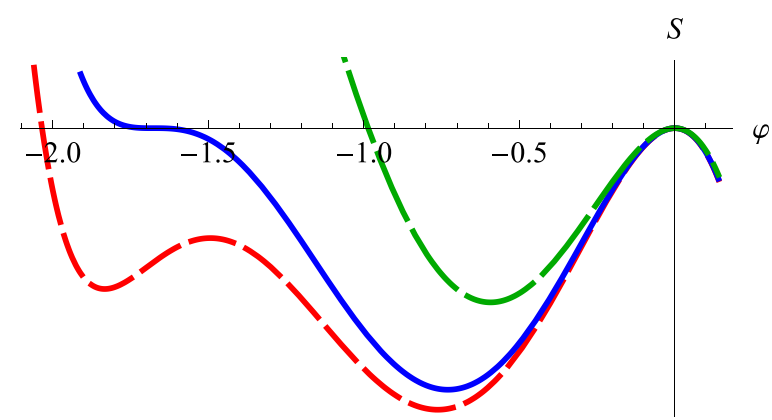

FIG. 14. Typical Sagdeev pseudopotentials having a negative triple root structure (solid blue curve, at $M / M_{s}=1.0390$ ), a supersoliton (red dashes, at $M / M_{s}=1.0397$ ), and an ordinary soliton (long green dashes, at $M / M_{s}=1.0352$ ), all three for $f=0.786, \beta=0$, and $\mu=1$.

we pick $g=0.9$ and $\mu=1$, and then $f=0.786$ and $M /$ $M_{s}=1.0390$ ensure that a triple root exists, at $\varphi=-1.677$. The corresponding Sagdeev pseudopotential is indicated in Fig. 14 by the solid blue line. By increasing $M / M_{s}$ above the triple root value $M / M_{s}=1.0390$, supersolitons are obtained, and by decreasing $M / M_{s}$ below this, standard solitons are generated, for the same plasma composition. The corresponding solitary structure and electric field profiles have not been included here, as they are analogous to those illustrating Sec. III.

Because of the many papers in the literature using Boltzmann hot species, there is no point to further dwell on this limit, once it has been amply shown that triple root structures can be generated.

\section{SUMMARY}

We have revisited a specific dusty plasma model having nonthermal Cairns electrons and ions, and cold, negative and positive dust grains. Aspects of large-amplitude dust-acoustic solitons and double layers had been investigated before, with an emphasis on their existence ranges and generic properties, ${ }^{16}$ but preceding the introduction of new concepts like supersolitons, solitons at the acoustic speed, and even acoustic speed supersolitons. It was suggested for a very specific plasma composition and soliton speed that three distinct roots of the same polarity might coalesce into a triple root.

In this paper, therefore, rather than the incidental attention obtained so far, a systematic investigation has been carried out into the existence domains and properties of triple root structures. Because the Sagdeev pseudopotential is, from a mathematical point of view, continuous in the different compositional parameters, Mach numbers, and the electrostatic potential, there is a wide range where triple root structures can exist. Triple root structures are shown to be intimately connected to double layer and supersoliton ranges, as the nonthermal parameters are changed, rather than merely the normalized soliton speed or Mach number. This shows that triple root structures can be much more common than at first expected, even at the acoustic speed or in the presence of Boltzmann rather than Cairns nonthermal hot species.

Once the existence domains are properly established, one can work out as many examples in detail as one chooses. We have tested a great many combinations of compositional 
parameters, and they all reveal a similar pattern, with quantitative but no qualitative changes. None of these have been included in the presentation, to avoid overloading the paper with repetitive graphs and figures, except for a couple of typical, generic examples, selected among an infinity of other equally valid choices with analogous results.

This type of analysis has then been extended to triple root structures at the acoustic speed and to the regime where the hot species are Boltzmann, which is one of the limits of the nonthermal Cairns description.

Finally, we have on purpose taken the same nonthermality parameter $\beta$ and the same temperature $T$ for both the Cairns nonthermal hot electrons and ions, for the simple reason that otherwise the parametric discussion becomes unwieldy and cannot be carried out in a rather comprehensive and systematic way. The main argument is that if, with this simplification, triple root structures and supersolitons can be generated, a more complex model will certainly also contain these, as one has more compositional parameters to play with. Experience has shown that the more complicated the composition the more soliton modes can be obtained, but we feel that with double layers, triple root structures, supersolitons, and coexistence regimes we have quite a rounded off picture.

Indeed, to the best of our knowledge, we have not encountered in the literature or among our own efforts examples of physically relevant Sagdeev pseudopotentials having five local extrema of the same polarity outside equilibrium. These would, if existing, allow the theoretical possibility of having two successive double layers for a judicious choice of compositional parameters and structure speeds.

\section{ACKNOWLEDGMENTS}

F.V. thanks the Centre for Space Research (North-West University, Potchefstroom) for its warm hospitality on a previous visit during which this work originated. C.O. wishes to acknowledge the financial assistance of the National Research Foundation (NRF) towards this research. The opinions expressed and conclusions arrived at are those of the authors and are not necessarily to be attributed to the NRF.

${ }^{1}$ F. Verheest, Waves in Dusty Space Plasmas (Kluwer, Dordrecht, 2000).

${ }^{2}$ P. K. Shukla and A. A. Mamun, Introduction to Dusty Plasma Physics (IOP Press, London, 2002).

${ }^{3}$ V. W. Chow, D. A. Mendis, and M. Rosenberg, J. Geophys. Res. 98, 19065, https://doi.org/10.1029/93JA02014 (1993).
${ }^{4}$ D. A. Mendis and M. Rosenberg, Annu. Rev. Astron. Astrophys. 32, 419 (1994).

${ }^{5}$ M. Horányi, Annu. Rev. Astron. Astrophys. 34, 383 (1996).

${ }^{6}$ N. N. Rao, P. K. Shukla, and M. Y. Yu, Planet. Space Sci. 38, 543 (1990).

${ }^{7}$ F. Verheest, Planet. Space Sci. 40, 1 (1992).

${ }^{8}$ A. Barkan, R. L. Merlino, and N. D’Angelo, Phys. Plasmas 2, 3563 (1995).

${ }^{9}$ F. Verheest, Space Sci. Rev. 77, 267 (1996).

${ }^{10}$ P. H. Sakanaka and P. K. Shukla, Phys. Scr. T84, 181 (2000).

${ }^{11}$ A. A. Mamun and P. K. Shukla, Geophys. Res. Lett. 29(18), 17-1-17-4, https://doi.org/10.1029/2002GL015219 (2002).

${ }^{12}$ I. Spassovska and P. H. Sakanaka, in Magnetic Fields in the Universe: From Laboratory and Stars to Primordial Structures, edited by E. M. de Gouveia dal Pino, G. Lugones, and A. Lazarian (American Institute of Physics, New York, 2005), pp. 506-520.

${ }^{13}$ N. Akhtar, S. Mahmood, and H. Saleem, Phys. Lett. A 361, 126 (2007).

${ }^{14}$ A. A. Mamun, Phys. Lett. A 372, 686 (2008).

${ }^{15}$ F. Sayed, M. M. Haider, A. A. Mamun, P. K. Shukla, B. Eliassson, and N. Adhikary, Phys. Plasmas 15, 063701 (2008).

${ }^{16}$ F. Verheest, Phys. Plasmas 16, 013704 (2009).

${ }^{17}$ A. E. Dubinov and D. Y. Kolotkov, IEEE Trans. Plasma Sci. 40, 1429 (2012).

${ }^{18}$ A. E. Dubinov and D. Y. Kolotkov, Plasma Phys. Rep. 38, 909 (2012).

${ }^{19}$ A. Das, A. Bandyopadhyay, and K. P. Das, J. Plasma Phys. 78, 149 (2012).

${ }^{20}$ F. Verheest, M. A. Hellberg, and I. Kourakis, Phys. Plasmas 20, 012302 (2013).

${ }^{21}$ F. Verheest, M. A. Hellberg, and I. Kourakis, Phys. Rev. E 87, 043107 (2013).

${ }^{22}$ F. Verheest, M. A. Hellberg, and I. Kourakis, Phys. Plasmas 20, 082309 (2013).

${ }^{23}$ M. A. Hellberg, T. K. Baluku, F. Verheest, and I. Kourakis, J. Plasma Phys. 79, 1039 (2013).

${ }^{24}$ F. Verheest, G. S. Lakhina, and M. A. Hellberg, Phys. Plasmas 21, 062303 (2014).

${ }^{25}$ S. V. Singh and G. S. Lakhina, Commun. Nonlinear Sci. Numer. Simul. 23, 274 (2015)

${ }^{26}$ F. Verheest and S. R. Pillay, Phys. Plasmas 15, 013703 (2008).

${ }^{27}$ F. Verheest and S. R. Pillay, Nonlinear Proc. Geophys. 15, 551 (2008).

${ }^{28}$ F. Verheest, Phys. Plasmas 17, 062302 (2010).

${ }^{29}$ F. Verheest and M. A. Hellberg, Phys. Plasmas 17, 102312 (2010).

${ }^{30}$ T. K. Baluku, M. A. Hellberg, and F. Verheest, Europhys. Lett. 91, 15001 (2010).

${ }^{31}$ F. Verheest, Phys. Plasmas 18, 083701 (2011).

${ }^{32}$ F. Verheest and M. A. Hellberg, Phys. Plasmas 22, 012301 (2015).

${ }^{33}$ R. Z. Sagdeev, Reviews of Plasma Physics, edited by M. A. Leontovich (Consultants Bureau, New York, 1966), Vol. 4, p. 23.

${ }^{34}$ R. A. Cairns, A. A. Mamun, R. Bingham, R. Boström, R. O. Dendy, C. M. C. Nairn, and P. K. Shukla, Geophys. Res. Lett. 22, 2709, https://doi.org/ 10.1029/95GL02781 (1995).

${ }^{35}$ J. F. McKenzie, Phys. Plasmas 9, 800 (2002).

${ }^{36}$ F. Verheest, T. Cattaert, G. S. Lakhina, and S. V. Singh, J. Plasma Phys. 70, 237 (2004)

${ }^{37}$ T. K. Baluku and M. A. Hellberg, Phys. Plasmas 19, 012106 (2012).

${ }^{38}$ F. Verheest, M. A. Hellberg, and T. K. Baluku, Phys. Plasmas 19, 032305 (2012).

${ }^{39}$ C. P. Olivier, F. Verheest, and S. K. Maharaj, J. Plasma Phys. 83, 905830605 (2017) 